CLAWAR 2020: $23^{\text {rd }}$ International Conference on

Climbing and Walking Robots and the Support

Technologies for Mobile Machines,

Moscow, Russian Federation, 24-26 August 2020.

https://doi.org/10.13180/clawar.2020.24-26.08.55

\title{
THE EXPERIMENTAL INVESTIGATION OF THE SENSITIVITY IN THE EXOSKELETON CONTROL LOOP
}

\author{
V. G. GRADETSKY, I. L. ERMOLOV, M. M. KNYAZKOV, E. A. SEMENOV and A. N. \\ SUKHANOV \\ Institute for Problems in Mechanics of the Russian Academy \\ of Sciences 101-1, Prospect Vernadskogo, 119526 Moscow, \\ Russiasukhanov-artyom@yandex.ru,
}

\begin{abstract}
An exoskeleton system contains a human operator in the control loop, which imposes restrictions on the applied control algorithms and movement speed. Thus, the creation of exoskeleton device with an EMG-based control system that takes into account the interaction of humans and exoskeleton design elements becomes one of the most actual tasks of modern robotics.

At the moment, there are a number of tasks in research projects towards exoskeleton control algorithms. These tasks include consideration of fatigue of a person arising from the control of the exoskeleton over long period of time. Operator's fatigue, as a result of the monotonous operations, leads to the fact that the control efficiency decreases, and the positioning error will increase over time. Another task when controlling using human biopotentials is compensation of the influence of the operator's tremor on the control signal. Also, a very important factor is the adaptation of actuators to a change in the transient characteristics of external and internal forces. The designed control algorithms should prevent possible injuries while using the exoskeleton device.

This article describes the results of tests of an arm exoskeleton device with DC drive located in the elbow joint and a control algorithm based on an electromyogram of the biceps brachii and triceps brachii of the operator. The structure and features of the experimental stand developed in the laboratory of robotics and mechatronics of IPMech RAS are shown. The features of parameter settings during calibration of the exoskeleton control system are shown.
\end{abstract} Sensitivity.

Key words: Exoskeleton, Electromyogram, Control, Experiment, Muscles, Motor control,

\section{Introduction}

Developing a control system for an active exoskeleton with use the operator's electromyogram as the setting signal has its own distinctive features. Unlike bilateral systems, the operator is strictly linked to the slave system of the exoskeleton, which imposes restrictions on movement speed and acceleration [1]. The control algorithms for such type of exoskeleton device should include consideration of tremor and fatigue to ensure safety in control.

The relevance of the research is related to the need of improving the quality of control in the human-machine system, due to the adaptation of the control system to the psychophysiological characteristics of the certain operator. Determining the critical parameters of the electromyogram readings during training and performing technological operations will allow one to develop effective algorithms for adapting the control system to the influence of external and internal factors on control [2]. The control system needs adjusting parameters to take into account the appearance of a new operator, or adjust these parameters in the conditions of monotonous work and the appearance of signs of fatigue. For example, in [3], the authors describe modern evaluation methods for muscle fatigue based on the surface EMG data analysis in statics and dynamics. The authors showed that continuous monitoring of local muscle fatigue when performing certain physical work is possible by measuring the myoelectric activity of individual muscles using surface electromyography methods. The practical application of such techniques in relation to an exoskeleton device, the control system of which is based on the use 
of information about muscle biopotentials, is the possibility of creating an algorithm for configuring the exoskeleton control system for a specific user and for a specific task type.

In previous works, researchers have proved that muscle fatigue is expressed in a decrease in frequency and an increase in the amplitude of the electromyogram signal $[4,5,11]$. Thus, it is important to take into account the individual characteristics of each skeletal muscle involved in control. These features are expressed in the variation of geometry [6], force and velocity of contraction dependences [13], conductivity of the skin [14-15], the age of the operator [7], the level of its muscle tone $[12,16]$, and so on. Therefore, when developing an exoskeleton control system, it is reasonable to create special databases for different groups of operators with similar characteristics. Based on the obtained characteristics, one can develop a set of values for each group, which will serve as a reference when calibrating the control system.

\section{The experimental stand specification}

Earlier in our work [8,9], we performed mathematical modeling of the interaction between the exoskeleton and the operator. These theoretical studies have shown the importance of the operation mode (speed mode, compensation of external forces mode when holding an object).

This paper presents the creation of a working model of the elbow exoskeleton device and presents the results of experimental studies conducted in the laboratory of robotics and mechatronics of the Institute for problems in mechanics of the Russian Academy of Sciences. The technical scheme of the designed stand is shown on the Figure 1.

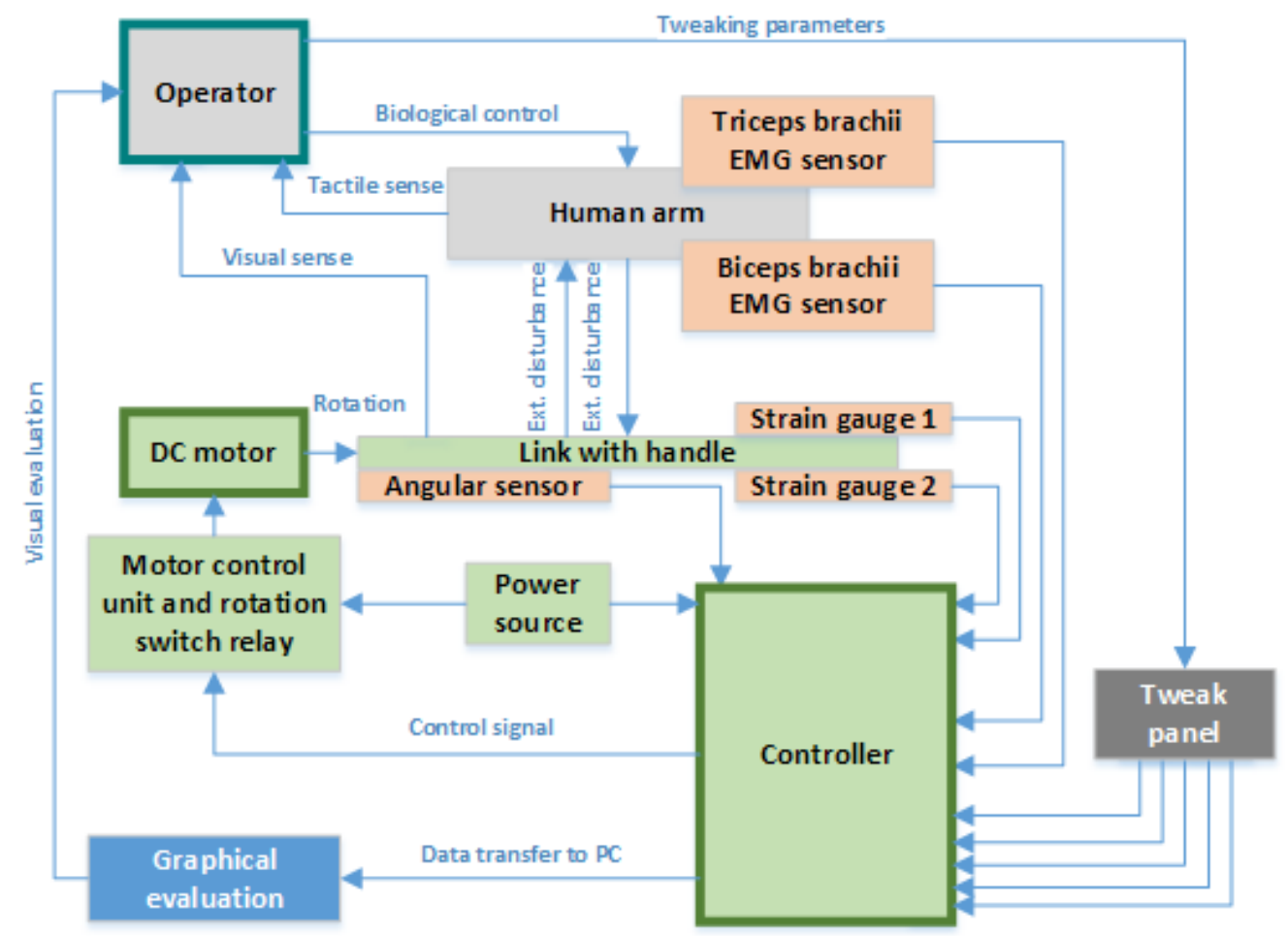

Fig. 1. The technical scheme of the system.

The experimental stand contains a control unit that receives information about the current position of the exoskeleton elements in space and the state of activity of the operator's muscle groups. The exoskeleton link, driven by a DC motor, is equipped with power sensors that 
measure the reaction forces between the structural element of the exoskeleton and the operator's arm. The operator use visual and tactile channels to evaluate the motion.

In our research, we used two DC motors. One of the DC motors was equipped with a reversible gear box so that the operator could feel the reaction forces while holding the load in isometric operation. Another DC motor was equipped with a worm gear to simulate motion blocking when implementing an algorithm for determining the maximum force parameter from various operators.

To evaluate the reaction forces, we used two strain gauges integrated into the exoskeleton handle with a maximum payload of $500 \mathrm{~N}$, as well as a HX711 ADC unit with a resolution of 24 bits and $80 \mathrm{~Hz}$ measurement frequency (Fig.2). To tune control parameters, a control panel with a set of variable resistors was implemented, which allows correcting the transmitted values according to the proposed algorithms.
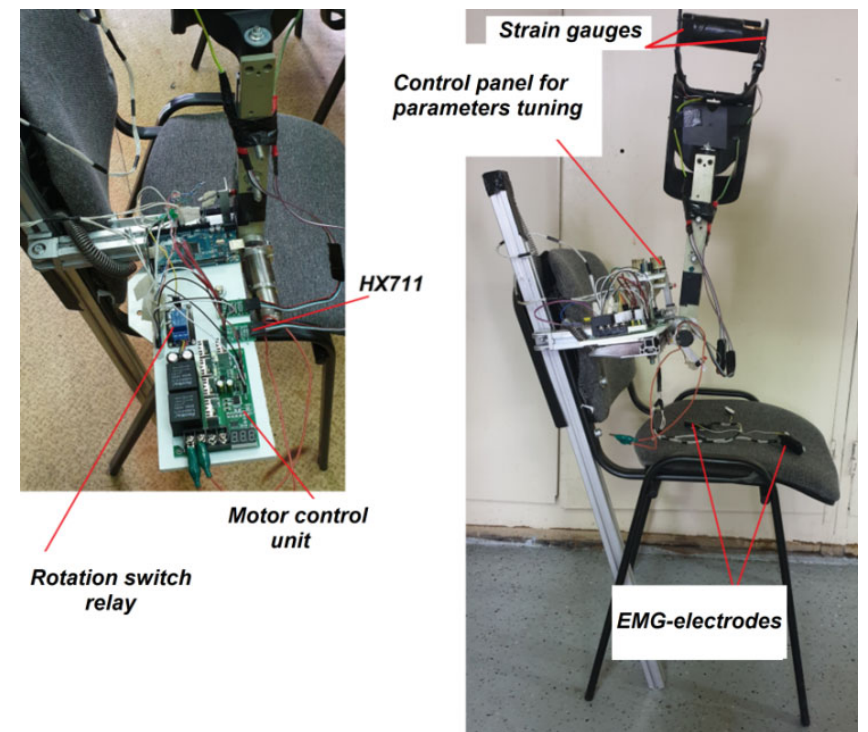

Fig. 2. General view of the experimental stand at the final stage of development

To obtain data on the muscle activity, Myoware Muscle Sensors AT-04-001 were used. Their applicability for the obtaining an electromyogram of muscles was discussed in paper [10]. In this work, we used raw signal from these sensors to improve performance and apply our own software filter with dynamic adjustment of filtration parameters. For processing the obtained data and form the control signal via PWM we used a controller based on the Atmega2560 RISC processor with a clock frequency of $16 \mathrm{MHz}$ and a 10-bit ADC.

\section{The experimental results}

On the base of the laboratory we conducted several groups of experimental studies. A group of 3 test subjects was created. The first experiment was to find out the difference in EMG behavior between 3 subjects in simple motion. The total electromyogram data in this experiment was the readings of the potential difference on the biceps brachii $E M G_{b i c}$ and on the triceps brachii $E M G_{\text {tric }}$.

$$
E M G_{\text {sum }}=E M G_{\text {bic }}+E M G_{\text {tric }}
$$


Within the control program, the obtained data of the triceps brachii $E M G_{t r i c}$ was taken with a negative sign in order to determine the direction of movement of the exoskeleton link from the resulting sign of the total data $E M G_{\text {sum }}$.

Figure 3 shows the results of an experiment on bending and unbending the arm at the elbow with a weight of $3 \mathrm{~kg}$ for each of the subjects. In this experiment, the behavior of an electromyogram without filtering was evaluated.

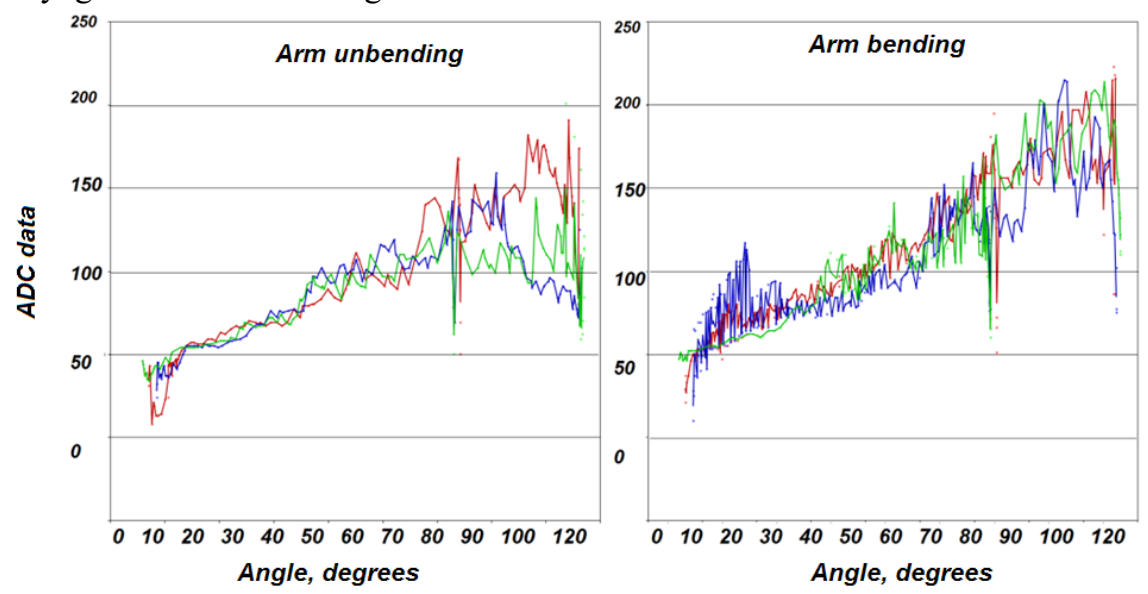

Fig. 3. Raw total EMG data of the biceps and triceps brachii

Here one can see a tendency of increasing the EMG amplitude with an increase in the angle in the elbow joint.

This effect can be explained that arm compensates the gravity of the payload with the most active biceps brachii. As shown by further experiments, to obtain a negative value of the parameter $E M G_{\text {sum }}$, which means that when activating the reverse rotating of the exoskeleton's motor, it is necessary to take into account the position of the arm in space, as well as the individual characteristics of the muscles used. From this we can conclude that value of the parameter $E M G_{\text {sum }}$ must be corrected.

$$
E M G_{\text {sum }}=a * E M G_{\text {bic }}+b * E M G_{\text {tric }}
$$

Here, variables $a$ and $b$ are functions that depend on the maximum effort that a given muscle can develop. Maximum forces for each muscle were obtained in the experiment via strain gauges. In the control system, these parameters are used as settings for the sensitivity of sensors to changes in the electromyogram amplitude. Table 1 shows the results of numerical adjustment of parameters $a$ and $b$ for each test subject in the group when setting the movement at a uniform speed.

Table 1. The results of the settings

\begin{tabular}{|c|c|c|}
\hline & Parameter $a$ & Parameter $b$ \\
\hline Subject 1 & 0,861 & 1,074 \\
\hline Subject 2 & 0,911 & 1,268 \\
\hline Subject 3 & 0,927 & 0,983 \\
\hline Average in the group & 0,899 & 1,108 \\
\hline
\end{tabular}

Changes in the amplitude of the graphs obtained in this experiment indicate the presence of noise that needs to be filtered. Therefore, the next group of experiments was devoted to configuring the noise filter [17] for the parameter $E M G_{\text {sum }}$ entering the control system. For this 
experiment, the moving average algorithm was selected. This algorithm allows filtering out low frequencies. Implementing this filter in the control system requires setting two parameters - the time constant and the filtration coefficient. The value of the filtered parameter $E M G_{\text {sum }}$ will depend on the parameter value on the current and subsequent sensor polling cycle:

$$
E M G_{\text {sum } . k}=E M G_{\text {sum } . k} *(1-N)+E M G_{\text {sum } . k+1} * N
$$

Here the parameter $\mathrm{N}=[0 \ldots 1]$ is the filtration coefficient. Increasing the value of this parameter leads to the return of the influence of noise on the received signal; however, as it was shown by experimental studies, a high value of this parameter reduces the sensitivity of the control system. If the filtration coefficient is high, it is difficult for the operator to make a sudden movement or change the direction of movement. The time constant specifies the time interval during which averaging will occur. An increase in the time constant leads to smoother changes in the parameter $E M G_{\text {sum }}$, but introduces a noticeable delay in control. In the course of experiments, it was found that increasing the time constant parameter is suitable for slow movements characteristic of the exoskeleton load holding mode or for improving positioning accuracy. Sudden movements that are typical, for example, for fast technological operations, require reducing this coefficient.

As part of the experiment, the subjects were given the task to bend and unbend the arm at the elbow in an exoskeleton while holding loads of different weights and at the same time observe a constant speed. The speed constancy was controlled in the developed program. During the experiment, the settings for the filter time constant $\mathrm{T}$ and the filter coefficient $\mathrm{n}$ were changed. When the flexion-extension speed was constant for five approaches, the current parameters were recorded in the database. In this case, the angular range of 10 degrees, set aside for acceleration and deceleration near the extreme positions defined by limit switches, was not taken into account. Table 2 shows the final values of the parameters after the experiment.

Table 2 . The results of the settings

\begin{tabular}{|c|c|c|c|c|}
\hline & \multicolumn{2}{|c|}{$\begin{array}{l}\text { Fast movements ( } 1 \text { second to } \\
\text { overcome } 100 \text { degrees) }\end{array}$} & \multicolumn{2}{|c|}{$\begin{array}{l}\text { Slow movement ( } 4 \text { seconds to } \\
\text { overcome } 100 \text { degrees) }\end{array}$} \\
\hline & Parameter $T$ & Parameter $N$ & Parameter $T$ & Parameter $N$ \\
\hline \multicolumn{5}{|l|}{ Subject 1} \\
\hline $1 \mathrm{~kg}$ & $2 \mathrm{~ms}$ & 0,05 & $4 \mathrm{~ms}$ & 0,1 \\
\hline $3 \mathrm{~kg}$ & $2 \mathrm{~ms}$ & 0,05 & $5 \mathrm{~ms}$ & 0,1 \\
\hline $5 \mathrm{~kg}$ & $3 \mathrm{~ms}$ & 0,04 & $5 \mathrm{~ms}$ & 0,08 \\
\hline \multicolumn{5}{|l|}{ Subject 2} \\
\hline $1 \mathrm{~kg}$ & $2 \mathrm{~ms}$ & 0,05 & $4 \mathrm{~ms}$ & 0,1 \\
\hline $3 \mathrm{~kg}$ & $2 \mathrm{~ms}$ & 0,05 & $5 \mathrm{~ms}$ & 0,1 \\
\hline $5 \mathrm{~kg}$ & $2 \mathrm{~ms}$ & 0,05 & $5 \mathrm{~ms}$ & 0,09 \\
\hline \multicolumn{5}{|l|}{ Subject 3} \\
\hline $1 \mathrm{~kg}$ & $2 \mathrm{~ms}$ & 0,06 & $5 \mathrm{~ms}$ & 0,1 \\
\hline $3 \mathrm{~kg}$ & $3 \mathrm{~ms}$ & 0,05 & $5 \mathrm{~ms}$ & 0,09 \\
\hline $5 \mathrm{~kg}$ & $3 \mathrm{~ms}$ & 0,05 & $5 \mathrm{~ms}$ & 0,08 \\
\hline $\begin{array}{l}\text { Average in the } \\
\text { group }\end{array}$ & $2 \mathrm{~ms}$ & 0,05 & $5 \mathrm{~ms}$ & 0,09 \\
\hline
\end{tabular}

From this table, one can see that increasing the weight of the load leads to the need of increasing the value of the filter time constant to obtain a constant speed of movement of the 
exoskeleton link. This can be explained by the fact that the increased load on the biceps led to the involvement of more motor units per unit of time, which led to an amplification of the EMG signal and an increase in the amplitude of noise, which had to be filtered by increasing the value of the time constant of the filter. When performing the movement in slow mode, the load on the biceps lasted longer, so the effect of fatigue is visible here, expressed in a decrease in frequency and an increase in the amplitude of the useful signal.

The next group of studies was aimed at identifying the value of the dead zone for the assigned biopotential. Experiments have shown that even in the state of rest and noise filtering, the value of $E M G_{\text {sum }}$ will be different from zero, and since this value is used to form the motor speed, constant small deviations of the rotor from the current position are possible in the state of rest. Therefore, the speed calculation algorithm has been adjusted to take into account the dead zone parameter, which is configured individually for each operator.

The final experiments were devoted to checking the values of the control system parameters calibrated according to the found standards for the fourth untrained test subject. A load of $5 \mathrm{~kg}$ was placed on the handle of the test stand, and the task of moving at a constant speed was set. Here are screenshots of the program written in "Processing" that represent interface to obtain data from experimental stand (Fig. 4, 5):

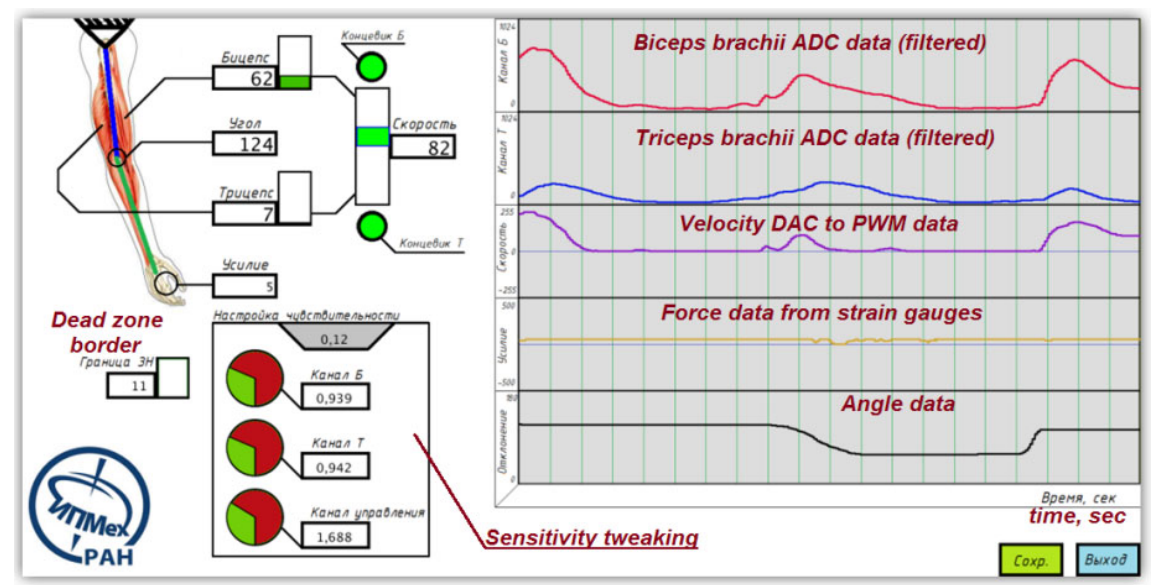

Fig. 4. The result of an experiment with a fourth subject with a calibrated control system when implementing slow movements.

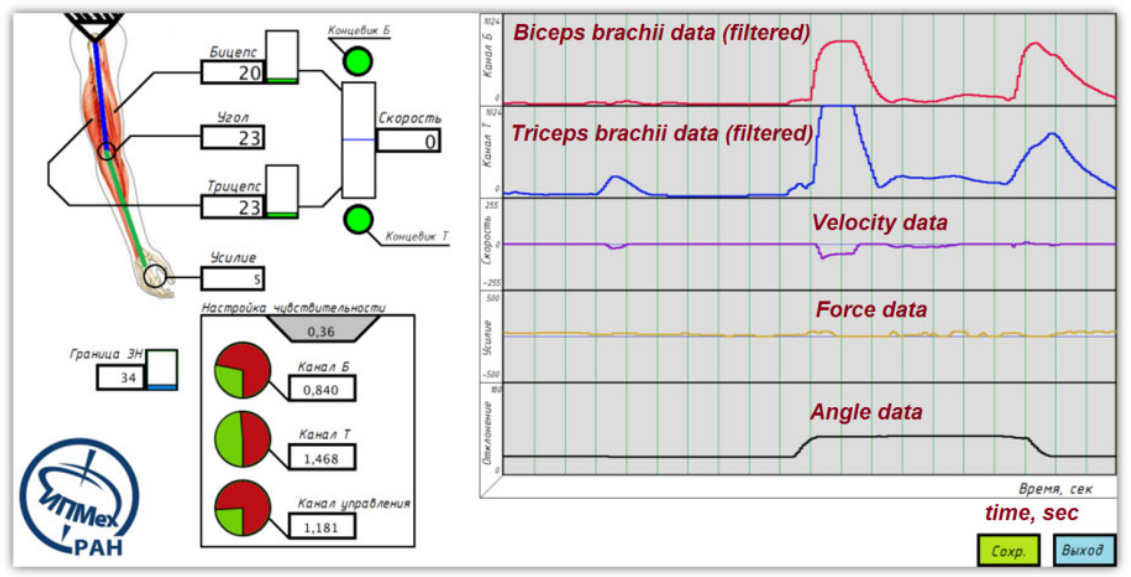

Fig. 5. The result of an experiment with a fourth subject with a calibrated control system when implementing fast movements. 


\section{Conclusion}

In the course of experimental studies, the influence of various parameters of the control system on the quality of movement control of the exoskeleton device was shown. The experimental stand and special software were developed to allow flexible configuration of the control system parameters. As part of the work, various data were obtained for several operators, on the basis of which reference values of control system parameters were obtained for calibration. The results of testing the developed algorithm on full-scale stand, simulating the arm exoskeleton with the DC drive, located in the elbow joint and controlling algorithms based on the pattern envelope electromyogram of the biceps brachii and triceps brachii of the operator were presented. The structure and features of the stand developed in the laboratory of robotics and mechatronics of IPMech RAS are shown. A comparative characteristic of the quality of control of the DC drive that is part of an exoskeleton, on which the proposed algorithm for configuring the control system in relation to one operator when changing to another was worked out. The following control quality indicators were evaluated - over-regulation (when evaluating the speed), time to set the specified position (when setting the filter coefficients and determining the numeric value of the dead zone parameter), and the accuracy of positioning the control point on the exoskeleton link.

\section{Acknowledgments}

The present work was supported by the Ministry of Science and Higher Education within the framework of the Russian State Assignment under contract No. AAAA-A20-120011690138-6.

\section{References}

1. N.N. Rukina, A.N. Kuznetsov, V.V. Borzikov, O.V. Komkova, A.N. Belova, Surface electromyography: its role and potential in the development of exoskeleton (review). Sovremennye tehnologii v medicine 2016; 8(2): 109-118, http://dx.doi.org/10.17691/ stm2016.8.2.15.

2. R. Verdugo, J. Matamala, (2019). Clinical Neurophysiology Standards of EMG instrumentation: Twenty years of changes. Clinical Neurophysiology. 131. 10.1016/j.clinph.2019.08.023.

3. M. Cifrek, V.Medved, S. Tonkovic, S. Ostojić, (2009). Surface EMG Based Muscle Fatigue Evaluation in Biomechanics. Clinical biomechanics (Bristol, Avon). 24. 327-40. 10.1016/j.clinbiomech.2009.01.010.

4. N.A. Dimitrova, G.V. Dimitrov, 2003. Interpretation of EMG changes with fatigue: facts, pitfalls, and fallacies. J. Electromyogr. Kines. 13 (1), 13-36.

5. C.J. De Luca, 1992. Spectral compression of the EMG signal as an index of muscle fatigue. In: Sargeant, A.J., Kernell, D. (Eds.), Neuromuscular Fatigue. Royal Netherlands Academy of Arts and Sciences, Amsterdam, The Netherlands, pp. 44-51.

6. V. De Sapio, (2014). An approach for goal-oriented neuromuscular control of digital humans in physics-based simulations. Int. J. of Human Factors Modelling and Simulation. 4. 121 - 144. 10.1504/IJHFMS.2014.062387.

7. V. Valderrabano, B.M. Nigg, B. Hintermann, B. Goepfert, W. Dick, C.B. Frank, V. von. Tscharner, (2007). Muscular Lower Leg Asymmetry in Middle-Aged People. Foot \& Ankle International, 28(2), 242-249. https://doi.org/10.3113/FAI.2007.0242

8. V. G. Gradetsky, I. L. Ermolov, M. M. Knyazkov, E. A. Semenov, and A. N. Sukhanov. Switching operation modes algorithm for the exoskeleton device. In Smart Electromechanical Systems, volume 261 of Studies in Systems, Decision and Control, pages 131-142. Springer Nature Switzerland, 2020.

9. V. Gradetsky, I. Ermolov, M. Knyazkov, E. Semenov, and A. Sukhanov. Features of human-exoskeleton interaction. In Studies in Systems, Decision and Control, volume 261 
of Robotics: Industry 4.0 Issues \& New Intelligent Control Paradigms, pages 77-88. Springer Nature Switzerland Switzerland, 2020.

10. S. Fuentes, S. Santos-Cuadros, E. Olmeda, C. Álvarez-Caldas, V. Díaz, J. San Román, (2019). Is the Use of a Low-Cost sEMG Sensor Valid to Measure Muscle Fatigue?. Sensors. 19. 3204. 10.3390/s19143204.

11. S. Andersson, Active Muscle Control in Human Body Model Simulations, Master's Thesis in Automotive Engineering, CHALMERS, Applied Mechanics, Master's Thesis 2013: 62, P. 64

12. F.E. Zajac and M.E. Gordon, "Determining muscle's force and action in multi-articular movement," Exerc. Sport Sci. Rev, vol. 17, pp. 187-230, 1989. and Zajac, F.E. (1989) Muscle and tendon: properties, models, scaling, and application to biomechanics and motor control. CRC Critical Reviews in Biomedical Engineering 17, 359-411.

13. J. L. Sancho-Bru, A. Pérez-González, M. C. Mora, B. E. León, M. Vergara, J. L. Iserte, P. J. Rodríguez-Cervantes, and A. Morales, "Towards a realistic and self-contained biomechanical model of the hand," 2011.

14. D.R. Wilkie, The mechanical properties of muscle, British Medical Bulletin, 1956, V. 12.

15. V.S. Abbott, D.R. Wilkie, the relation between velocity of shortening and the tension-length curve of skeletal muscle, Journal of Physiology, 1953, V. 120

16. V.S. Novoselov, "On mathematical models of molecular contraction of skeletal muscles", Vestnik SPbGU. Ser. 10. 2016. Vol. 3, p. 88-96 (in Russian)

17. F. Sado, H.J. Yap, R.A.R. Ghazilla, N. Ahmad (2018) Exoskeleton robot control for synchronous walking assistance in repetitive manual handling works based on dual unscented Kalman filter. PLoS ONE 13(7): e0200193. https://doi.org/10.1371/journal.pone.0200193 